\section{(6) OPEN ACCESS}

\title{
Altered breathing mechanics and ventilatory response during exercise in children born extremely preterm
}

\author{
J E MacLean, ${ }^{1,2}$ K DeHaan, ${ }^{1}$ D Fuhr, ${ }^{3}$ S Hariharan, ${ }^{1,2}$ B Kamstra, ${ }^{1,2}$ L Hendson, ${ }_{1}^{4}$ \\ I Adatia, ${ }_{1}^{1,2}$ C Majaesic, ${ }^{1}$ A T Lovering, ${ }^{5}$ R B Thompson, ${ }^{6}$ D Nicholas, $^{7}$ B Thebaud, ${ }^{8}$ \\ M K Stickland ${ }^{2}$
}

\begin{abstract}
- Additional material is published online only. To view please visit the journal online (http://dx.doi.org/10.1136/ thoraxjnl-2015-207736).
\end{abstract}

For numbered affiliations see end of article.

\section{Correspondence to} Dr Joanna E MacLean, Division of Respiratory Medicine, Department of Pediatrics, University of Alberta, 4-590 Edmonton Clinic Health Academy, 11405 87th Avenue, Edmonton, Alberta, Canada, T6G 1C9; joanna.maclean@ualberta.ca

Received 23 August 2015 Revised 25 April 2016 Accepted 3 May 2016 Published Online First 3 June 2016

\section{CrossMark}

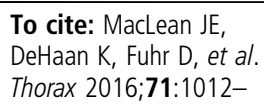

1019.

\section{ABSTRACT \\ Background Extreme preterm birth confers risk of long-term impairments in lung function and exercise capacity. There are limited data on the factors contributing to exercise limitation following extreme preterm birth. This study examined respiratory mechanics and ventilatory response during exercise in a large cohort of children born extremely preterm (EP). \\ Methods This cohort study included children 8- 12 years of age who were born EP ( $\leq 28$ weeks gestation) between 1997 and 2004 and treated in a large regionalised neonatal intensive care unit in western Canada. EP children were divided into no/mild bronchopulmonary dysplasia (BPD) (ie, supplementary oxygen or ventilation ceased before 36 weeks gestational age; $n=53$ ) and moderate/severe BPD (ie, continued supplementary oxygen or ventilation at 36 weeks gestational age; $n=50$ ). Age-matched control children $(n=65)$ were born at full term. All children attempted lung function and cardiopulmonary exercise testing measurements.}

Results Compared with control children, EP children had lower airway flows and diffusion capacity but preserved total lung capacity. Children with moderate/ severe BPD had evidence of gas trapping relative to other groups. The mean difference in exercise capacity (as measured by oxygen uptake $\left(\mathrm{VO}_{2}\right) \%$ predicted) in children with moderate/severe BPD was $-18 \pm 5 \%$ and $-14 \pm 5.0 \%$ below children with no/mild BPD and control children, respectively. Children with moderate/ severe BPD demonstrated a potentiated ventilatory response and greater prevalence of expiratory flow limitation during exercise compared with other groups. Resting lung function did not correlate with exercise capacity.

Conclusions Expiratory flow limitation and an exaggerated ventilatory response contribute to respiratory limitation to exercise in children born EP with moderate/ severe BPD.

\section{INTRODUCTION}

Children born preterm have a significant risk for long-term impairment of respiratory and cardiovascular function. Infants at imminent risk for early respiratory impairment, including bronchopulmonary dysplasia (BPD), are those born extremely preterm (EP; $\leq 28$ weeks gestational age, GA)

\section{Key messages}

What is the key question?

- What is the relationship between lung function and limitation to exercise in children born extremely preterm (EP)?

What is the bottom line?

- Expiratory flow limitation and an exaggerated ventilatory response contribute to respiratory limitation to exercise in subgroups of children born EP.

\section{Why read on?}

- Despite lung function impairments in children born EP with no/mild and moderate/severe bronchopulmonary dysplasia, only those with moderate/severe bronchopulmonary dysplasia demonstrate impaired exercise capacity, altered breathing mechanics and heightened ventilatory response during exercise compared with control children.

during the late canalicular stage of lung development when airways and lung vasculature come together. ${ }^{1}$ The impact of EP birth on lung development is fewer and larger alveoli, loss of small pulmonary arteries, lower capillary density and altered airway mechanics. ${ }^{1}$ Follow-up studies of children born preterm in the surfactant era and studied in middle childhood or adolescence document reduced airway flows, lung diffusion impairment and increased gas trapping compared with control children. $^{2-6}$ While some studies document greater levels of lung function impairment in children with a history of BPD, ${ }^{245}$ compared with preterm birth alone, others show limited or no differences between preterm children with and without a history of BPD. ${ }^{3}{ }^{6}$ Exercise capacity, measured by peak oxygen uptake $\left(\mathrm{VO}_{2 \text { peak }}\right)$, may be impaired in children born preterm. ${ }^{7-9}$ Limited data are available on how impaired lung function in children born preterm alters exercise capacity and breathing mechanics during exercise.

Exercise impairments in children born preterm, with and without a history of BPD, are attributed 
to ventilatory limitation despite few studies reporting ventilatory data during exercise. These studies report higher oxygen uptake for the same power output in children with BPD, ${ }^{10}$ impaired $^{11}$ and enhanced ${ }^{12}$ soluble gas transfer in response to exercise in children with BPD and young adults born preterm, respectively, and altered breathing mechanics during exercise in children born preterm ${ }^{7}$ and children with BPD $^{10}$ compared with control children with little information on the mechanisms behind these changes. Expiratory flow limitation (EFL) may develop during exercise in children with a history of preterm birth $;^{7}$ however, EFL and operating lung volumes during exercise have not previously been examined.

The aim of this study was to investigate respiratory mechanics and ventilatory response during exercise in a large cohort of children born EP in the era of routine antenatal steroids and surfactant use. We hypothesised that children born EP will show impairments in $\mathrm{VO}_{2 \text { peak }}$ proportionate to reduction in lung function and reduced airway flows during exercise relative to control children. Understanding the determinants of reduced exercise capacity is an important step towards reducing the risk of long-term cardiorespiratory complications of EP birth.

\section{METHODS}

\section{Subjects}

This single-centre cohort study included children born EP ( $\leq 28$ weeks GA) who were alive at 18 months of age; children diagnosed with non-ambulatory cerebral palsy or legal blindness at the 18 month assessment were excluded because of potential physical limitations to completing exercise testing. This definition of EP is the current minimal standard for follow-up in Canada, varies from that of WHO ( $<28$ weeks GA; http://www. who.int/mediacentre/factsheets/fs363/en/) but is consistent with the GA recommendation of the National Institute of Child Health and Human Development (NICHD).$^{13}$ EP children were cared for at a large regionalised tertiary care centre in western Canada between 1997 and 2004. Children born EP were categorised as no/mild BPD or moderate/severe (mod/sev) BPD using the definitions of the NICHD/National Heart, Lung, Blood Institute (NHLBI)/Office of Rare Diseases (ORD) workshop. ${ }^{14}$ This corresponded to the definition of BPD used in the neonatal intensive care unit (NICU) at the time of these admissions of supplementary oxygen use at 36 weeks GA such that infants off supplementary oxygen at 36 weeks would have no/ mild BPD and those using supplementary oxygen or ventilation, $\mathrm{mod} / \mathrm{sev}$ BPD. There was insufficient information available for further retrospective subclassification. Controls were born at term ( $\geq 37$ weeks GA) and had no significant history of cardiorespiratory disease based on parental report. Controls could have a history of mild asthma if this was well controlled ${ }^{15}$ at the time of the study. Controls were recruited as friends of EP children, and through posters as well as word of mouth within the university/hospital community. The Health Research Ethics Board approved this study. Additional details of the protocol are included in the online supplement.

\section{Study protocol}

Families who agreed to participate were mailed a package of questionnaires and scheduled for an activity day. On the activity day, informed consent and assent were obtained from the parent/guardian and child, respectively. The activity day included lung function testing and cardiopulmonary exercise testing (CPET). A subset of children completed echocardiography at rest. Medical chart review was completed for children born EP to collect the NICU data. GA and birth weight for controls were obtained by parent report.

\section{Ethnicity, anthropometry, cardiorespiratory health and activity}

Ethnicity was identified for each parent. Children with parents of different ethnicities were coded as mixed ethnicity. Height, weight and body mass index (BMI) were converted to z-scores using available normative data (http://www.cdc.gov/ growthcharts/cdc_charts.htm). Children were deemed to have a history of asthma if parents reported a history of asthma at enrolment or on questionnaires. Illness history, respiratory symptoms and medication use for the last 12 months were collected by parent report. Activity was assessed using questions from the Child Behaviour Checklist; parents were asked to rate how much time their child spent in each sport/physical activity compared with other children (less than average, average, more than average, don't know). All children rated as spending 'more than average' time in any sport/physical activity were considered to be active more than average.

\section{Lung function testing}

Lung function testing was performed according to published criteria. ${ }^{16-18}$ Testing was completed using a mass flow sensor and plethysmography (Vmax series; SensorMedics Corporation, Yorba Linda, California, USA). Measured spirometry values were converted to z-scores using Global Lung Initiative equations. ${ }^{19}$ Published reference equations for Caucasian children were used for lung volumes and diffusion capacity (transfer factor) with equations for residual volume (RV)/total lung capacity (TLC) obtained through personal communication with the author. $^{20}$

\section{Cardiopulmonary exercise testing}

An incremental exercise test was performed on a cycle ergometer. A ramped exercise protocol was used with the workload increments selected $(5-20 \mathrm{~W} / \mathrm{min})$ based on the child's predicted peak power output such that peak oxygen consumption would be obtained in 10-12 min of exercise. Breath-by-breath measurements were collected by a metabolic cart (Vmax Spectra V29 system; SensorMedics, Yorba Linda, California, USA). Satisfactory participant effort was confirmed based on one or more of the following criteria: (1) plateau in oxygen consumption; (2) respiratory quotient (RQ) $>1.15$; (3) patient exhaustion; or (4) evidence of a ventilatory limitation to exercise. ${ }^{21}$ Results were expressed as $\mathrm{VO}_{2 \text { peak }}(\mathrm{L} / \mathrm{min})$ and $\mathrm{VO}_{2 \text { peak }}$ relative to body weight $(\mathrm{L} / \mathrm{min} / \mathrm{kg}) . \mathrm{VO}_{2 \text { peak }} \%$ predicted was calculated using published reference equations for children. ${ }^{22}$ Inspiratory capacity (IC) manoeuvres were conducted at rest, every 2 min and at peak exercise. EFL was present when the intersection of the exercising tidal volume $\left(\mathrm{V}_{\mathrm{T}}\right)$ loop and the maximal flow volume loop was greater than $5 \%{ }^{23}$ This was determined by a single blinded reviewer. Rating of perceived exertion (RPE) and dyspnoea were assessed using standard numerical scales. ${ }^{24}$ Maximal voluntary ventilation (MVV) was measured following published criteria; ${ }^{16} 40$ children were unable to successfully complete MVV measurement (26\%; 15 BPD, 5 EP, 20 control) and were excluded from analysis involving MVV. Breathing reserve $(\mathrm{BR})$ was calculated as $\mathrm{BR}=\left(\mathrm{MVV}-\mathrm{V}_{\text {Epeak }}\right) / \mathrm{MVV}$.

\section{Data management and analysis}

The study data were managed using REDCap electronic data capture tools. Statistical analyses were performed using IBM SPSS Statistic V.21 (IBM and others, 1989, 2013). $\chi^{2}$ and 
Goodman and Kruskal's $\gamma$ were used to test associations between groups and categorical or ordinal data, respectively. Student's t-tests and one-way analysis of variance (ANOVA) were used to compare continuous normally distributed outcome variables between groups. Multivariable linear regression models were developed for $\mathrm{VO}_{2 \text { peak }}$ with variables for inclusion selected based on univariable analysis where $\mathrm{p}<0.10$; perinatal variables were entered into the model only for EP children. A $\mathrm{p}<0.05$ indicated statistically significant effects with Bonferroni correction applied for post hoc comparisons.

\section{RESULTS}

\section{Study population}

A total of 357 children born EP met eligibility criteria for inclusion in the study and had GA, birth weight and BPD status available. Of these, 103 (29\%) consented to participate in this follow-up study. There were no differences with respect to GA ( $26.4 \pm 1.3$ weeks vs $26.4 \pm 1.3$ weeks, $\mathrm{p}=$ not significant (ns)), birth weight $(896 \pm 200 \mathrm{~g}$ vs $920 \pm 1.79 \mathrm{~g}, \mathrm{p}=\mathrm{ns})$ and the prevalence of mod/sev BPD (45\% vs 49\%, Pearson's $\chi^{2} 3.4$, $\mathrm{p}=\mathrm{ns})$ between participants and non-participants. Figure 1 shows the distribution of $\mathrm{mod} / \mathrm{sev}$ BPD, no/mild BPD and control children successfully completing lung function and CPET measurements. Children noted by parents to have physical or cognitive limitations were less likely to complete testing (see online supplement).

Perinatal data show that children with $\mathrm{mod} / \mathrm{sev}$ BPD were born earlier, and had lower birth weight than children with no/ mild BPD (table 1). Premature rupture of membranes, antenatal corticosteroids, clinical chorioamnionitis and respiratory distress syndrome did not differ between groups. Doses of surfactant, days of ventilation and NICU length of stay (LOS) were higher for $\mathrm{mod} / \mathrm{sev}$ BPD. Children with $\mathrm{mod} / \mathrm{sev}$ BPD were more likely to be born by caesarean section, and to receive postnatal corticosteroids as well as surgical management for patent ductus arteriosus than children with no/mild BPD.

There were no differences in sex and ethnicity frequencies between $\mathrm{mod} / \mathrm{sev} \mathrm{BPD}$, no/mild $\mathrm{BPD}$ and control children
Figure 1 Distribution of extremely preterm eligible and enrolled children as well as control children. The number of children successfully completing each activity day measure is shown. More children with $\mathrm{mod} / \mathrm{sev}$ BPD were unable to complete lung volume $(20 \%$ vs $5.7 \%$ vs $1.5 \%$, $\mathrm{p}<0.01)$, diffusion capacity $(26 \%$ vs $3.8 \%$ vs $4.6 . \%, \mathrm{p}<0.001)$ and CPET (14\% vs $1.9 \%$ vs $4.6 \%, p<0.05)$. BPD, bronchopulmonary dysplasia; CPET, cardiopulmonary exercise testing; mod/ sev, moderate/severe.

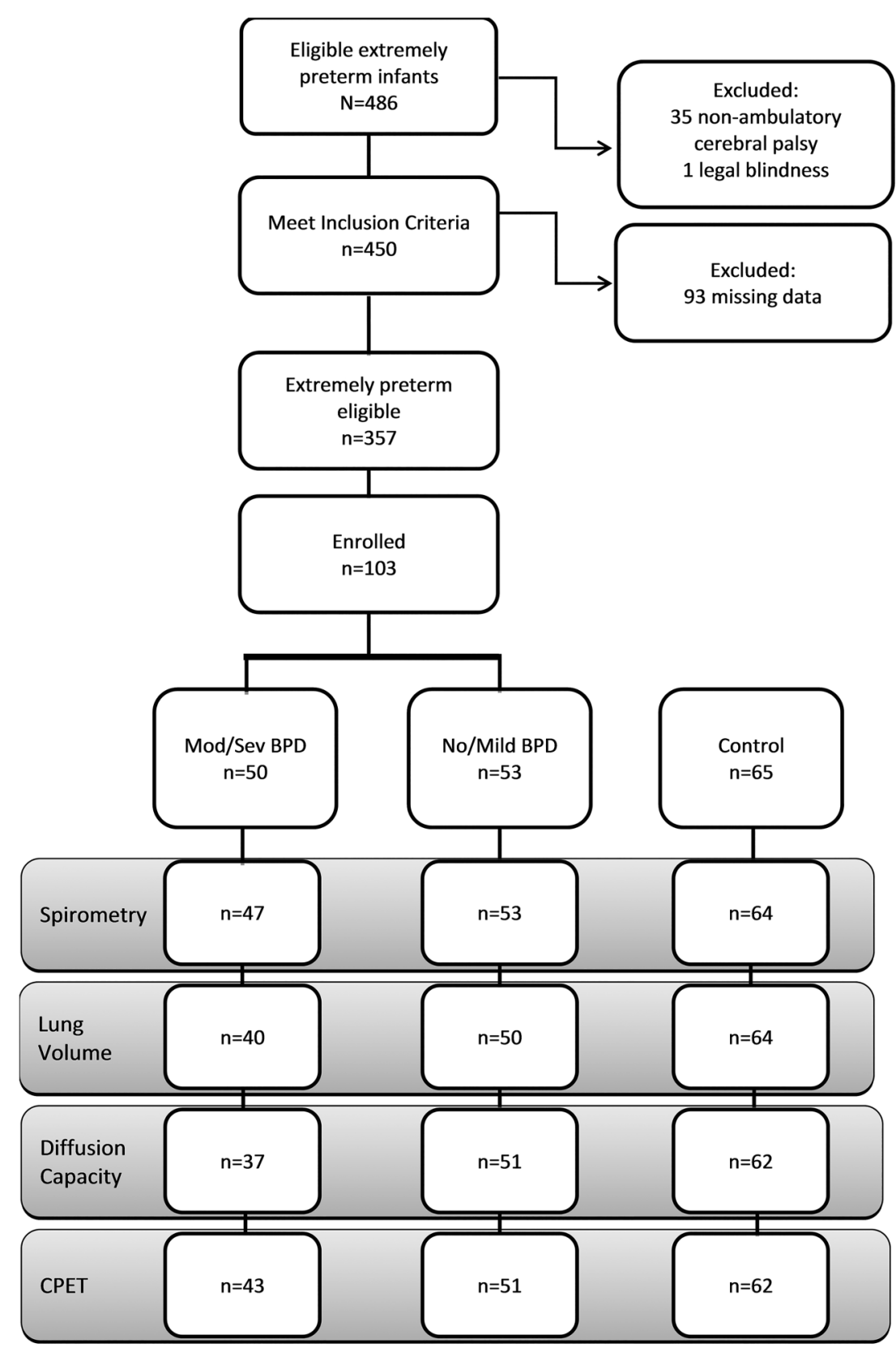


Table 1 Perinatal data for children born extremely preterm

\begin{tabular}{|c|c|c|}
\hline & $\begin{array}{l}\text { Moderate/severe BPD } \\
(\text { mean } \pm S D ; n(\%))\end{array}$ & $\begin{array}{l}\text { No/mild BPD } \\
(\text { mean } \pm S D ; n(\%))\end{array}$ \\
\hline $\mathrm{n}$ & 47 & 53 \\
\hline Gestational age (weeks)* & $26.0 \pm 1.9$ & $26.7 \pm 1.1$ \\
\hline Birth weight $(\mathrm{g}) \ddagger$ & $813 \pm 191$ & $982 \pm 163$ \\
\hline Maternal age (years) & $29.6 \pm 5.8$ & $30.3 \pm 6.3$ \\
\hline Doses of surfactant $t$ & $2.3 \pm 2.1$ & $1.1 \pm 1.8$ \\
\hline $\begin{array}{l}\text { Days of initial } \\
\text { ventilation } \neq\end{array}$ & $29 \pm 22$ & $6 \pm 11$ \\
\hline NICU LOS (days) $\ddagger$ & $104 \pm 32$ & $65 \pm 28$ \\
\hline PROM & $10(21 \%)$ & $7(13 \%)$ \\
\hline Antenatal corticosteroids & $33(70 \%)$ & $43(83 \%)$ \\
\hline Clinical chorioamnionitis & $3(6 \%)$ & $4(8 \%)$ \\
\hline Caesarean section* & $34(72 \%)$ & $27(52 \%)$ \\
\hline RDS & $45(98 \%)$ & $51(98 \%)$ \\
\hline PDA requiring surgery* & $12(27 \%)$ & $5(10 \%)$ \\
\hline Postnatal corticosteroids* & $13(28 \%)$ & $3(6 \%)$ \\
\hline
\end{tabular}

(table 2). Children with mod/sev BPD and no/mild BPD had lower height, weight and BMI z-scores than control children. Rates of asthma and wheeze in the last 12 months, but not asthma medication use, differed by group with the highest rates in children with $\mathrm{mod} / \mathrm{sev}$ BPD. A greater proportion of control children were reported to be more active than average and shortness of breath (SOB) with activity was more common in children with $\mathrm{mod} / \mathrm{sev}$ BPD.

\section{Lung function and echocardiography}

Lung function results differed between groups (table 3). $\mathrm{FEV}_{1}$, $\mathrm{FEV}_{1} / \mathrm{FVC}, \mathrm{FEF}_{25-75}$ were lower in $\mathrm{mod} / \mathrm{sev} \mathrm{BPD}$ and no/mild BPD compared with control children with no statistically significant differences between EP groups. The proportion of children with spirometric measures below the lower limit of normal differed by group with a higher proportion of children with $\mathrm{mod} / \mathrm{sev}$ BPD and no/mild BPD with abnormal results compared with control children (see online supplementary table E1). Lung volume measurements showed higher RV and RV/TLC in
Table 2 Demographics at follow-up for extremely preterm and control children

\begin{tabular}{|c|c|c|c|}
\hline & $\begin{array}{l}\text { Mod/sev BPD } \\
(\text { mean } \pm \text { SD, } \\
\text { n (\%)) }\end{array}$ & $\begin{array}{l}\text { No/mild BPD } \\
(\text { mean } \pm \text { SD, } \\
\text { n (\%)) }\end{array}$ & $\begin{array}{l}\text { Control } \\
(\text { mean } \pm S D, \\
n(\%))\end{array}$ \\
\hline$n$ & 47 & 53 & 64 \\
\hline Age (years) & $11.9 \pm 1.6$ & $12.3 \pm 1.5$ & $11.6 \pm 1.9$ \\
\hline Height z-score $\ddagger$ & $-0.27 \pm 0.98$ & $-0.07 \pm 1.05$ & $0.45 \pm 0.88$ \\
\hline Weight z-score $\ddagger$ & $-0.074 \pm 1.28$ & $-0.39 \pm 0.99$ & $0.45 \pm 0.85$ \\
\hline BMI z-scoret & $0.074 \pm 1.24$ & $-0.42 \pm 1.01$ & $0.33 \pm 1.04$ \\
\hline$\%$ Male & $27(57 \%)$ & $34(64 \%)$ & $37(58 \%)$ \\
\hline$\%$ Caucasian & $34(79 \%)$ & $40(82 \%)$ & $45(82 \%)$ \\
\hline $\begin{array}{l}\text { Maternal smoking in } \\
\text { pregnancy }\end{array}$ & $11(24 \%)$ & $10(19 \%)$ & $7(12 \%)$ \\
\hline $\begin{array}{l}\text { Household cigarette smoke } \\
\text { exposure }\end{array}$ & $15(32 \%)$ & $16(31 \%)$ & $12(20 \%)$ \\
\hline Asthma* & $18(38 \%)$ & $16(30 \%)$ & $11(17 \%)$ \\
\hline Wheeze in last 12 months* & $19(40 \%)$ & $13(24 \%)$ & $9(15 \%)$ \\
\hline $\begin{array}{l}\text { Asthma medications in last } \\
12 \text { months }\end{array}$ & $10(21 \%)$ & $7(13 \%)$ & $4(6 \%)$ \\
\hline Activity more than average $f$ & $15(32 \%)$ & $27(51 \%)$ & $37(63 \%)$ \\
\hline SOB with activity $\dagger$ & $18(38 \%)$ & $8(15 \%)$ & $9(15 \%)$ \\
\hline \multicolumn{4}{|c|}{ 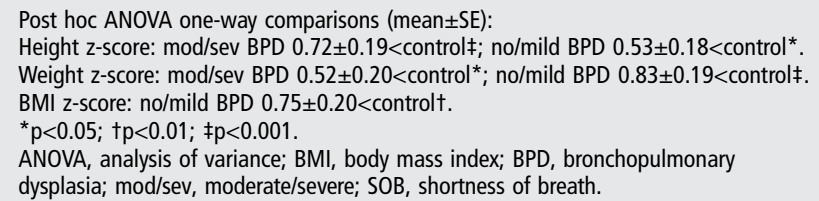 } \\
\hline
\end{tabular}

children with $\mathrm{mod} / \mathrm{sev}$ BPD compared with control children. zTLCO/alveolar volume was lower in both EP groups compared with control children. MVV did not differ between groups. No group differences were seen in routine clinical echocardiography results (see online supplementary table E2).

\section{Cardiopulmonary exercise testing}

Peak RQ and heart rate were similar between the three groups, indicating that all three groups achieved similar relative peak intensity during CPET (table 4). $\mathrm{VO}_{2 \text { peak }}$ relative, $\mathrm{VO}_{2} \%$ predicted and peak carbon dioxide uptake $\mathrm{VCO}_{2 \text { peak }}$ were lower in children with $\mathrm{mod} / \mathrm{sev}$ BPD compared with no/mild BPD and control children with no significant differences between children with no/mild BPD and control children (table 4). In the mod/sev

Table 3 Lung function tests comparing extremely preterm and control children

\begin{tabular}{|c|c|c|c|c|c|}
\hline & \multirow[b]{2}{*}{ Mod/sev BPD (mean \pm SD) } & \multirow[b]{2}{*}{ No/mild BPD (mean \pm SD) } & \multirow[b]{2}{*}{ Control $($ mean \pm SD) } & \multicolumn{2}{|l|}{ Differences from control } \\
\hline & & & & Mod/sev BPD (mean $(95 \% \mathrm{CI}))$ & No/mild BPD (mean $(95 \% \mathrm{Cl}))$ \\
\hline $\mathrm{zFEV}_{1}^{\ddagger}$ & $-0.92 \pm 1.36$ & $-0.50 \pm 1.10$ & $0.09 \pm 1.01$ & $-1.00(-1.54$ to -0.47$) \dagger$ & $-0.58(-1.10 \text { to }-0.068)^{*}$ \\
\hline zFVC & $-0.85 \pm 1.67$ & $0.35 \pm 1.25$ & $0.47 \pm 0.90$ & & \\
\hline $\mathrm{zFEV}_{1} / \mathrm{FVC} \dagger$ & $-1.14 \pm 1.5$ & $-1.3 \pm 0.80$ & $-0.57 \pm 1.14$ & $-0.56(-1.11$ to -0.015$) \dagger$ & $-0.71(-1.25$ to -0.19$) \dagger$ \\
\hline $\mathrm{zFEF}_{25-75} \ddagger$ & $-1.6 \pm 1.22$ & $-1.3 \pm 0.87$ & $-0.48 \pm 1.12$ & $-1.09(-1.59$ to -0.58$) \ddagger$ & $-0.84(-1.33$ to -0.35$) \ddagger$ \\
\hline zTLC & $0.63 \pm 1.08$ & $0.66 \pm 1.10$ & $0.38 \pm 1.02$ & & \\
\hline$z R V^{*}$ & $0.092 \pm 1.12$ & $-0.16 \pm 0.95$ & $-0.47 \pm 0.97$ & $0.56(0.70 \text { to } 1.06)^{*}$ & \\
\hline zRV/TLC $†$ & $-0.19 \pm 1.33$ & $-0.55 \pm 1.04$ & $-0.91 \pm 1.00$ & $0.72(0.18$ to 1.27$) \dagger$ & \\
\hline zFRC & $0.22 \pm 1.12$ & $0.072 \pm 0.92$ & $-0.29 \pm 1.11$ & & \\
\hline zTLCO & $-0.49 \pm 0.76$ & $-0.52 \pm 0.82$ & $-0.32 \pm 0.69$ & & \\
\hline zTLCO/VAt & $-1.64 \pm 0.89$ & $-1.57 \pm 0.76$ & $-1.06 \pm 0.95$ & $-0.58(-1.02$ to -0.13$) \dagger$ & $-0.50(-0.91$ to -0.10$) \dagger$ \\
\hline MVV (L) & $79.5 \pm 27.5$ & $83.3 \pm 27.9$ & $93.1 \pm 30.7$ & & \\
\hline
\end{tabular}

${ }^{*} \mathrm{p}<0.05 ; \mathrm{tp}<0.01 ; \neq \mathrm{p}<0.001$.

$\mathrm{BPD}$, bronchopulmonary dysplasia; $\mathrm{FEF}_{25-75}$, forced expiratory flow between $25-75 \%$ of FVC; FRC, functional residual capacity; mod/sev, moderate/severe; MVV, maximum voluntary ventilation; RV, residual volume; TLC, total lung capacity; TLCO, transfer factor for carbon monoxide; VA, alveolar volume; $z, z$-score. 
Table 4 Cardiopulmonary exercise test (CPET) cardiovascular responses at peak exercise comparing extremely preterm and control children

\begin{tabular}{|c|c|c|c|}
\hline & $\begin{array}{l}\text { Mod/sev BPD } \\
(\text { mean } \pm \text { SD) }\end{array}$ & $\begin{array}{l}\text { No/mild BPD } \\
(\text { mean } \pm S D)\end{array}$ & $\begin{array}{l}\text { Control } \\
(\text { mean } \pm \text { SD) }\end{array}$ \\
\hline Exercise time (min:sec) & $6: 52 \pm 2: 01$ & $8: 33 \pm 2: 12$ & $7: 45 \pm 1: 39$ \\
\hline RQ & $1.07 \pm 0.086$ & $1.07 \pm 0.84$ & $1.07 \pm 0.081$ \\
\hline Heart rate (beats/min) & $175.8 \pm 14.9$ & $178.1 \pm 19.1$ & $182.5 \pm 15.5$ \\
\hline Work (W) & $89.2 \pm 32.4$ & $104.5 \pm 33.2$ & $109.6 \pm 35.8$ \\
\hline $\mathrm{VO}_{2 \text { peak }}(\mathrm{L} / \mathrm{min})$ & $1.65 \pm 0.65$ & $1.83 \pm 0.55$ & $1.87 \pm 0.43$ \\
\hline $\mathrm{VO}_{2 \text { peak }} \%$ predicted $(\%) \dagger$ & $89.3 \pm 23.0$ & $107.4 \pm 22.7$ & $103.4 \pm 26.5$ \\
\hline $\mathrm{VO}_{2 \text { peak }}$ relative $(\mathrm{mL} / \mathrm{kg} / \mathrm{min}) \dagger$ & $36.9 \pm 10.7$ & $44.6 \pm 9.2$ & $42.6 \pm 9.6$ \\
\hline $\mathrm{VCO}_{2 \text { peak }}(\mathrm{L} / \mathrm{min})^{*}$ & $1.71 \pm 0.62$ & $1.98 \pm 0.65$ & $2.03 \pm 0.49$ \\
\hline
\end{tabular}

Post hoc ANOVA one-way comparisons (mean \pm SE).

VO ${ }_{2 \text { peak }}$ relative: $\mathrm{mod} / \mathrm{sev}$ BPD $5.73 \pm 1.94 \mathrm{~L} / \mathrm{min}<$ control $^{*}$; mod/sev BPD 7.72 $\pm 2.02 \mathrm{~L} / \mathrm{min}<$ no/mild BPDt.

$\mathrm{VO}_{2 \text { peak }} \%$ predicted: $\mathrm{mod} / \mathrm{sev}$ BPD $14 \pm 5 \%<$ control*; mod/sev BPD $18 \pm 5 \%<$ no/mild BPDt.

$\mathrm{VCO}_{2}$ peak: No statistically significant one-way differences.

${ }^{*} \mathrm{p}<0.05 ; \mathrm{p} p<0.01$.

ANOVA, analysis of variance; BPD, bronchopulmonary dysplasia; mod/sev, moderate/ severe; $\mathrm{RQ}$, respiratory quotient; $\mathrm{VCO}_{2 \text { peak, }}$ peak carbon dioxide uptake; $\mathrm{VO}_{2}$, oxygen uptake.

BPD group, $\mathrm{VO}_{2} \%$ predicted was $18.2 \pm 5.0 \%$ (mean $\pm \mathrm{SE}$ ) lower than children with no/mild BPD and 14.2 $\pm 4.9 \%$ (mean \pm SE) lower than control children.

\section{Breathing mechanics during exercise}

Minute ventilation $\left(\mathrm{V}_{\mathrm{E}}\right) / \mathrm{kg}$ at peak exercise was lower in children with $\mathrm{mod} / \mathrm{sev}$ BPD compared with no/mild BPD (table 5), with the difference attributable to lower $\mathrm{V}_{\mathrm{T}} / \mathrm{kg}$ in children with $\mathrm{mod} / \mathrm{sev}$ BPD. Oxygen saturation pulse $\left(\mathrm{SpO}_{2}\right)$ was maintained through exercise in all groups, and there was no between-group difference in $\mathrm{SpO}_{2}$ at baseline or peak exercise. All three groups demonstrated a comparable hyperventilatory response at peak exercise with similar end tidal carbon dioxide tension $\left(\mathrm{P}_{\mathrm{ET}} \mathrm{CO}_{2}\right)$ between groups. $\mathrm{V}_{\mathrm{E}} / \mathrm{VCO}_{2}$ slope was greater in the $\mathrm{mod} / \mathrm{sev}$ BPD group as compared with controls but similar in children with no/mild BPD and controls. The greater $\mathrm{V}_{\mathrm{E}} / \mathrm{VCO}_{2}$ slope in $\mathrm{mod} / \mathrm{sev} \mathrm{BPD}$ despite similar $\mathrm{P}_{\mathrm{ET}} \mathrm{CO}_{2}$ supports greater dead space ventilation in $\mathrm{mod} / \mathrm{sev} \mathrm{BPD}$.

BR was lower in children with no/mild BPD compared with controls though similar in children with mod/sev BPD compared with controls (table 5). EFL at peak exercise was more common in children with $\mathrm{mod} / \mathrm{sev}$ BPD as compared with no/mild BPD and controls. No group showed evidence of dynamic hyperinflation as IC was not reduced at peak exercise compared with baseline in any group. RPE and dyspnoea at peak exercise did not differ between groups.

\section{Multivariable model for $\mathrm{VO}_{2 \text { peak }}$}

Perinatal, demographic and lung function variables were tested to identify univariable predictors of $\mathrm{VO}_{2 \text { peak }} \%$ predicted. $\mathrm{BPD}$ group (mod/sev BPD, no/mild BPD, control), age and sex were included a priori. An additional 10 variables were identified on univariable analysis and entered into the initial model: 5 perinatal variables (antenatal corticosteroids, doses of surfactant, days of initial ventilation, postnatal steroids, NICU LOS), household cigarette smoke exposure, zWeight, zBMI, 'SOB with activity' and 'active more than average'. All of the identified perinatal variables correlated with BPD group and each other and, therefore, were removed from the model. zWeight and

Table 5 Cardiopulmonary exercise test (CPET) peak respiratory responses comparing extremely preterm (EP) children and control children

\begin{tabular}{|c|c|c|c|}
\hline & Mod/sev BPD (mean \pm SD) & No/mild BPD (mean $\pm S D)$ & Control $($ mean \pm SD) \\
\hline RR baseline (breaths/min)* & $23.0 \pm 4.6$ & $20.2 \pm 4.7$ & $20.6 \pm 5.2$ \\
\hline RR peak (breaths/min) & $48.0 \pm 12.8$ & $46.9 \pm 10.9$ & $47.5 \pm 12.4$ \\
\hline $\mathrm{V}_{\mathrm{T}} / \mathrm{kg}$ baseline $(\mathrm{mL} / \mathrm{kg})^{*}$ & $12.2 \pm 4.0$ & $14.9 \pm 6.3$ & $13.4 \pm 5.1$ \\
\hline $\mathrm{V}_{\mathrm{T}} / \mathrm{kg}$ peak $(\mathrm{mL} / \mathrm{kg}) \dagger$ & $25.7 \pm 7.5$ & $31.9 \pm 5.8$ & $29.5 \pm 6.5$ \\
\hline $\mathrm{V}_{\mathrm{E}} / \mathrm{kg}$ baseline $(\mathrm{mL} / \mathrm{kg} / \mathrm{min})$ & $266 \pm 101$ & $275 \pm 91$ & $250 \pm 90$ \\
\hline$V_{E} / k g$ peak $(\mathrm{mL} / \mathrm{kg} / \mathrm{min})^{*}$ & $1393 \pm 428$ & $1631 \pm 431$ & $1433 \pm 378$ \\
\hline Inspiratory capacity baseline (L) & $1.75 \pm 0.56$ & $1.74 \pm 0.74$ & $1.67 \pm 0.58$ \\
\hline Inspiratory capacity peak (L) & $1.84 \pm 0.47$ & $1.85 \pm 0.68$ & $1.94 \pm 0.68$ \\
\hline $\mathrm{SpO}_{2}$ baseline (\%) & $97.5 \pm 2.9$ & $97.7 \pm 2.7$ & $98.3 \pm 1.2$ \\
\hline $\mathrm{SpO}_{2}$ peak $(\%)$ & $94.3 \pm 4.9$ & $95.2 \pm 4.7$ & $95.3 \pm 4.9$ \\
\hline $\mathrm{P}_{\mathrm{ET}} \mathrm{O}_{2}$ peak $(\mathrm{mm} \mathrm{Hg})$ & $103.0 \pm 4.8$ & $103.8 \pm 4.9$ & $102.1 \pm 10.1$ \\
\hline $\mathrm{P}_{\mathrm{ET}} \mathrm{CO}_{2}$ peak $(\mathrm{mm} \mathrm{Hg})$ & $35.6 \pm 4.1$ & $35.6 \pm 3.9$ & $36.1 \pm 3.8$ \\
\hline $\mathrm{V}_{\mathrm{E}} / \mathrm{VO}_{2}$ slope & $27.9 \pm 3.3$ & $27.4 \pm 2.7$ & $26.4 \pm 2.8$ \\
\hline $\mathrm{V}_{\mathrm{E}} / \mathrm{VCO}_{2}$ slope s $^{*}$ & $30.5 \pm 3.8$ & $28.9 \pm 2.9$ & $28.6 \pm 3.2$ \\
\hline $\mathrm{BR}(\%)^{*}$ & $18.5 \pm 23.6$ & $15.5 \pm 20.9$ & $24.6 \pm 16.9$ \\
\hline Expiratory flow limitation $(n, \%)^{*}$ & $16 / 34(47 \%)$ & $16 / 49(33 \%)$ & $14 / 59(24 \%)$ \\
\hline End exercise-dyspnoea & $4.0 \pm 2.8$ & $4.4 \pm 2.2$ & $3.8 \pm 2.4$ \\
\hline End exercise-RPE & $6.0 \pm 2.5$ & $5.6 \pm 2.4$ & $5.0 \pm 2.7$ \\
\hline
\end{tabular}

${ }^{*} p<0.05$.

$t p<0.001$.

Post hoc ANOVA one-way comparisons (mean \pm SE).

RR baseline: $\mathrm{mod} / \mathrm{sev}$ BPD $2.8 \pm 1.0$ breaths $/ \mathrm{min}>$ no/mild BPD*

$\mathrm{V}_{\mathrm{T}} / \mathrm{kg}$ baseline: $\mathrm{mod} / \mathrm{sev} \mathrm{BPD} 2.7 \pm 1.1 \mathrm{~mL} / \mathrm{kg}<\mathrm{no} / \mathrm{mild} \mathrm{BPD}^{*}$.

$\mathrm{V}_{\mathrm{T}} / \mathrm{kg}$ peak: $\mathrm{mod} / \mathrm{sev}$ BPD $6.2 \pm 1.4 \mathrm{~mL} / \mathrm{kg}<\mathrm{no} / \mathrm{mild}$ BPDt; $\mathrm{mod} / \mathrm{sev}$ BPD $3.8 \pm 1.3 \mathrm{~mL} / \mathrm{kg}<$ control*; no/mild EP $3.8 \pm 1.3 \mathrm{~mL} / \mathrm{kg}>\mathrm{control}^{*}$.

$\mathrm{V}_{\mathrm{E}} / \mathrm{kg}$ peak: $\mathrm{mod} / \mathrm{sev}$ BPD $233 \pm 88 \mathrm{~mL} / \mathrm{kg} / \mathrm{min}<$ no/mild $B P D^{*}$.

$\mathrm{V}_{\mathrm{E}} / \mathrm{NCO}_{2}$ peak: $\mathrm{mod} / \mathrm{sev} \mathrm{BPD} 1.94 \pm 0.69<$ Control $^{*}$.

BR: no/mild BPD $9.0 \pm 3.8 \%<$ control* $^{*}$

ANOVA, analysis of variance; $\mathrm{BPD}$, bronchopulmonary dysplasia; $\mathrm{BR}$, breathing reserve; mod/sev, moderate/severe, $\mathrm{P}_{\mathrm{ET}} \mathrm{CO}_{2}$, end tidal carbon dioxide tension; $\mathrm{P}_{\mathrm{ET}} \mathrm{O}_{2}$, end tidal oxygen

tension; $\mathrm{RPE}$, rating of perceived exertion; $\mathrm{RR}$, respiratory rate; $\mathrm{SpO}_{2}$, oxygen saturation pulse; $\mathrm{VCO}_{2}$, carbon dioxide uptake; $\mathrm{V}_{\mathrm{E}}$, minute ventilation; $\mathrm{VO} \mathrm{O}_{2}$, oxygen uptake; $\mathrm{V}_{\mathrm{T}}$, tidal volume. 
Table 6 Predictors of exercise capacity as measured by $\mathrm{VO}_{2} \%$ predicted

\begin{tabular}{lrrr}
\hline Variables & B \pm SE & $\begin{array}{l}\text { Standardised } \\
\text { coefficient }\end{array}$ & p Value \\
\hline Group* & $-17.21 \pm 6.02$ & -0.56 & 0.005 \\
Male sex & $-24.42 \pm 9.03$ & -0.48 & 0.008 \\
Shortness of breath with activity & $-9.63 \pm 4.01$ & -0.16 & 0.018 \\
More active than average & $10.69 \pm 3.41$ & 0.21 & 0.003 \\
Age & $-3.19 \pm 0.95$ & -0.22 & 0.001 \\
zBMI & $-10.06 \pm 1.43$ & -0.47 & $<0.001$ \\
Interaction: groupxsex & $15.17 \pm 4.04$ & 0.95 & $<0.001$ \\
\hline The $R^{2}$ for the model is $0.43, p<0.001$. & & \\
*Group: 1=mod/sev BPD, 2=no/mild BPD, 3=control. & & \\
BMI, body mass index; BPD, bronchopulmonary dysplasia; VO $_{2,}$ oxygen uptake; \\
z, z-score.
\end{tabular}

household cigarette smoke exposure were not significant predictors and were removed. Finally, group was tested for significant interactions with other predictor variables and these interactions were added to the model. The final model used the 'enter' method for covariate inclusion, six main effects and one interaction term (table 6; adjusted $\mathrm{R}^{2} 0.43, \mathrm{p}<0.001$ ). BPD group, male sex, 'SOB with activity', higher age and higher BMI predicted lower $\mathrm{VO}_{2}$ peak \% predicted. 'Active more than average' predicted higher $\mathrm{VO}_{2}$ peak $\%$ predicted. Post hoc analysis demonstrated that, after controlling for other variables, $\mathrm{VO}_{2}$ peak \% predicted was lower in girls with mod/sev BPD compared with boys and higher in no/mild BPD and control girls compared with boys.

\section{DISCUSSION}

The results from the present study confirm impaired lung function and exercise capacity in preadolescents after EP birth in an era of routine antenatal steroids and postnatal surfactant, and highlight breathing mechanics and ventilatory response contributions to exercise limitation. Compared with control children, $\mathrm{FEV}_{1}$ was approximately $1.0 \mathrm{z}$-score lower in children with $\mathrm{mod} / \mathrm{sev} \mathrm{BPD}$ and $0.5 \mathrm{z}$-score lower in children with no/mild BPD. TLC was preserved with impairments in diffusion capacity in both EP groups compared with controls. $\mathrm{VO}_{2 \text { peak }}$ was $18 \%$ and 14\% lower, respectively, in children with mod/sev BPD compared with children with no/mild BPD and control children. Our results provide the first data documenting the factors contributing to respiratory limitation to exercise in preadolescent children with a history of $\mathrm{mod} / \mathrm{sev}$ BPD. This includes greater prevalence of EFL that cannot be explained by airflow obstruction alone and a heightened ventilatory response to exercise.

\section{Expiratory flow limitation}

In highly trained athletes and adults with respiratory disease, EFL promotes dynamic hyperinflation and intrinsic positive end-expiratory pressure leading to functional impairment of inspiratory muscle strength, increased work of breathing and increased sensations of dyspnoea. ${ }^{25}$ With ongoing EFL, end-expiratory lung volumes are increased with a corresponding reduction in IC and inspiratory reserve volumes; this decrease in IC during exercise would limit the ability to increase $V_{T}$ and may increase dyspnoea. In the present study, we demonstrate a greater prevalence of EFL in children with a history of BPD without group differences in IC suggesting that dynamic hyperinflation was not present. While there was reduction in peak $\mathrm{V}_{\mathrm{T}}$ response to exercise in the children with BPD compared with control and EP children, there was no evidence of hypercapnia or hypoxaemia at peak exercise. A blunted $\mathrm{V}_{\mathrm{T}}$ at peak exercise has previously been reported in children with a history of EP birth, ${ }^{7}$ and may be a compensatory mechanism to reduce work of breathing in the presence of EFL. Despite evidence of altered respiratory mechanics, dyspnoea at peak exercise was not more prevalent in children with mod/sev BPD suggesting either dyspnoea was not sensed or that the standard Borg scale may not be appropriate for assessing dyspnoea in this group. There were, however, group differences with respect to reported activity patterns such that children with a history of $\mathrm{mod} / \mathrm{sev}$ BPD were less active than children with no/mild BPD and control children. EFL leading to respiratory limitation during exercise could contribute to a chronic reduction in physical activity which, over time, would lead to a reduction in aerobic fitness and, hence, a lower $\mathrm{VO}_{2 \text { peak }}$. Further work is needed to explore the role of EFL in exercise limitation after EP birth.

\section{Potentiated ventilatory response}

Ventilatory inefficiency during exercise, as measured by an increase in the $\mathrm{V}_{\mathrm{E}} / \mathrm{VCO}_{2}$ slope, is an important predictor of health outcomes in adult patients with a range of cardiorespiratory diseases. A potentiated ventilatory response to exercise has been reported in patients with mild COPD $^{26}$ and pulmonary arterial hypertension (PAH), and is predictive of mortality in $\mathrm{PAH} .{ }^{27}$ The potentiated ventilatory response in these conditions may be linked to cardiac or pulmonary vascular abnormalities leading to stimulation of receptors in the lung and high vascular pressures. ${ }^{28}$ In the present study, children with a history of mod/ sev BPD showed a greater $\mathrm{V}_{\mathrm{E}} / \mathrm{VCO}_{2}$ slope response to exercise with similar $\mathrm{P}_{\mathrm{ET}} \mathrm{CO}_{2}$ values throughout exercise compared with children with no/mild BPD and control children. Echocardiographic measures at rest did not differ between groups suggesting this difference is unlikely due to cardiac or pulmonary vascular effects. However, exercise induced pulmonary vascular responses cannot be excluded. This pattern of response is consistent with the exercise response described in preadolescents with cystic fibrosis, another form of obstructive lung disease, who had mild to moderate airway obstruction where the ratio of dead space ventilation to $\mathrm{V}_{\mathrm{T}}$ was higher than controls throughout exercise. ${ }^{29}$ It is important to note that exercise training and breathing training have been shown to lower the slope of $\mathrm{V}_{\mathrm{E}} / \mathrm{VCO}_{2}$ response to exercise ${ }^{30}$ and reduce $\mathrm{EFL}^{31}$ and, therefore, may be appropriate therapeutic strategies in children and adults with a history of BPD.

\section{Relationship between lung function and exercise capacity}

The relationship between lung function and exercise capacity differs by the type of lung disease with limited data in children. For example, lung function (ie, $\mathrm{FEV}_{1}$ ) and $\mathrm{VO}_{2 \text { peak }}$ are positively, though weakly, correlated in some adult obstructive lung disease $^{32} 33$ and cardiac conditions ${ }^{34}$ though poorly correlated in restrictive lung disease. ${ }^{32}$ In adolescents with asthma $\mathrm{FEV}_{1}$ has been shown to be unrelated to $\mathrm{VO}_{2 \text { peak }}{ }^{35}$ suggesting that factors other than $\mathrm{FEV}_{1}$ explain the reduced $\mathrm{VO}_{2 \text { peak }}$ in asthma. Consistent with prior studies of preterm birth, ${ }^{8}{ }^{86}$ the present study of children born EP showed no significant relationships between lung function and $\mathrm{VO}_{2 \text { peak }}$. Exploration of the contribution of deconditioning and exercise training to reductions in exercise capacity in children following EP birth is needed. 


\section{Potential impact on adult lung function and exercise}

Airway flows and vital capacity of the lung as well as $\mathrm{VO}_{2 \max }$ begin to decrease in early adulthood. ${ }^{37}{ }^{38}$ For young adults with a history of EP birth, the normal decline in lung function and maximal oxygen uptake beginning in their mid-20's may have greater and earlier consequences. In addition to decline from a lower peak, factors associated with EP and impaired lung function may predispose to accelerated decline in lung function: inflammation, infection and hyperoxia are thought to contribute to the pathogenesis of BPD and are also linked to exaggerated age-related decline in lung function. ${ }^{39}$ Studies of young adults with a history of BPD, born at the beginning of the surfactant era, have documented abnormalities in lung function, exercise impairments and early emphysema. ${ }^{36}{ }^{40}$ Together, these studies suggest the development of a chronic obstructive lung disease in early adulthood. In the present study, children born EP have impaired $\mathrm{FEV}_{1}$ relative to controls with a doubling of this impairment in children with a history of mod/sev BPD. Even if differences from controls remain stable during ageing, EP birth will confer a high risk of earlier respiratory and exercise limitations in adulthood.

\section{Limitations}

Our study measurements precluded inclusion of children unable to pedal a bike, and, therefore, cannot be generalised to children with major disability. In addition, children with mod/sev BPD were more likely to be unable to complete components of testing excluding their results from these analyses. Our results are likely biased towards children with better exercise capacity and, therefore, will underestimate overall impairments following EP birth. Of note, lung measurements in controls differed from the anticipated z-score of 0 suggesting that the reference equations used may not be completely appropriate for this population. Measurements of activity were collected by parents' report rather than by objective measurement; future studies including objective activity data will be important to determine whether activity patterns are a factor in exercise limitation in mod/sev BPD and whether prescribed activity is a meaningful therapy. The prevalence of EFL was determined by plotting the tidal flow-volume loops within the maximum flowvolume curve at baseline. As such, we did not account for any exercise-induced bronchodilation, nor did we correct for thoracic gas compression. Lastly, we did not assess changes in lung diffusion, perfusion or ventilation/perfusion matching during exercise. These factors should be considered in future studies given at least one report of impairments in gas transfer during exercise and recovery, despite comparable resting measurements, in children born preterm with BPD compared with preterm and term control children. ${ }^{11}$

\section{Summary}

In summary, preadolescents with a history of EP birth with no/ mild BPD and mod/sev BPD have lower lung function relative to controls. Mod/sev BPD is associated with a greater reduction in lung function and impairments in exercise capacity. A heightened ventilatory response to exercise and EFL impact exercise capacity in those children with a history of mod/sev BPD. Both lung function and exercise testing are needed to fully characterise respiratory function and limitations in children born EP. Future research efforts should focus on defining the mechanisms for exercise limitation with the goal of identifying therapeutic targets to improve long-term cardiorespiratory outcomes following EP birth.

\section{Author affiliations \\ ${ }^{1}$ Faculty of Medicine and Dentistry, Departments of Pediatrics, University of Alberta,} Edmonton, Alberta, Canada
${ }^{2}$ Faculty of Medicine and Dentistry, Women \& Children's Health Research Institute, University of Alberta, Edmonton, Alberta, Canada

${ }^{3}$ Faculty of Medicine and Dentistry, Departments of Medicine, University of Alberta, Edmonton, Alberta, Canada

${ }^{4}$ Department of Pediatrics, University of Calgary, Calgary, Alberta, Canada ${ }^{5}$ Department of Human Physiology, University of Oregon, Eugene, Oregon, USA

${ }^{6}$ Faculty of Medicine and Dentistry, Biomedical Engineering, University of Alberta, Edmonton, Alberta, Canada

${ }^{7}$ Faculty of Social Work, University of Calgary, Calgary, Alberta, Canada

${ }^{8}$ Department of Pediatrics, University of Ottawa, Ottawa, Ontario, Canada

Acknowledgements The authors thank Mark Rosenthal for providing the equations for RV/TLC predicted values. REDCap electronic data capture tools were hosted by the Women \& Children's Health Research Institute, University of Alberta, Edmonton, $A B$, Canada.

Contributors JEM, BK, LH, IA, CM, ATL, RBT, DN, BT and MKS contributed to the designed the study. JEM, KD, DF, SH, BK and IA contributed to data acquisition. JEM and KD contributed to data analysis. JEM, KD, DF, LH, IA, ATL and MKS contributed to interpretation of the work. All authors contributed to revision of the manuscript, provided final approval of the manuscript and are accountable for all aspects of this work.

Funding Alberta Health Services (AHS) Emerging Research Team Grant.

Competing interests None declared.

Ethics approval University of Alberta Human Research Ethics Board.

Provenance and peer review Not commissioned; externally peer reviewed.

Open Access This is an Open Access article distributed in accordance with the Creative Commons Attribution Non Commercial (CC BY-NC 4.0) license, which permits others to distribute, remix, adapt, build upon this work non-commercially, and license their derivative works on different terms, provided the original work is properly cited and the use is non-commercial. See: http://creativecommons.org/ licenses/by-nc/4.0/

\section{REFERENCES}

1 O'Reilly M, Sozo F, Harding R. Impact of preterm birth and bronchopulmonary dysplasia on the developing lung: long-term consequences for respiratory health. Clin Exp Pharmacol Physiol 2013:40:765-73.

2 Vom Hove M, Prenzel F, Uhlig HH, et al. Pulmonary outcome in former preterm, very low birth weight children with bronchopulmonary dysplasia: a case-control follow-up at school age. J Pediatr 2014;164:40-5.

3 Cazzato S, Ridolfi L, Bernardi F, et al. Lung function outcome at school age in very low birth weight children. Pediatr Pulmonol 2013;48:830-7.

4 Hacking DF, Gibson AM, Robertson C, et al. Respiratory function at age 8-9 after extremely low birthweight or preterm birth in Victoria in 1997. Pediatr Pulmonol 2013:48:449-55

5 Vollsæter $\mathrm{M}$, Røksund $\mathrm{OD}$, Eide GE, et al. Lung function after preterm birth: development from mid-childhood to adulthood. Thorax 2013;68:767-76.

6 Malmberg LP, Pelkonen AS, Malmström K, et al. Very low birth weight and respiratory outcome: association between airway inflammation and hyperresponsiveness. Ann Allergy Asthma Immunol 2013;111:96-101.

7 Welsh L, Kirkby J, Lum S, et al. The EPICure study: maximal exercise and physical activity in school children born extremely preterm. Thorax 2010;65:165-72.

8 Clemm H, Røksund O, Thorsen E, et al. Aerobic capacity and exercise performance in young people born extremely preterm. Pediatrics 2012;129:e97-105.

9 Vrijlandt EJ, Gerritsen J, Boezen HM, et al. Lung function and exercise capacity in young adults born prematurely. Am J Respir Crit Care Med 2006;173:890-6.

10 Kriemler $\mathrm{S}$, Keller $\mathrm{H}$, Saigal $\mathrm{S}$, et al. Aerobic and lung performance in premature children with and without chronic lung disease of prematurity. Clin I Sport Med 2005;15:349-55.

11 Mitchell SH, Teague WG. Reduced gas transfer at rest and during exercise in school-age survivors of bronchopulmonary dysplasia. Am I Respir Crit Care Med 1998;157(Pt 1):1406-12.

12 Narang I, Bush A, Rosenthal M. Gas transfer and pulmonary blood flow at rest and during exercise in adults 21 years after preterm birth. Am J Respir Crit Care Med 2009;180:339-45.

13 Vohr B, Wright L, Hack M, et al. Follow-up care of high risk infants. Pediatrics 2004;114:1377-97.

14 Jobe AH, Bancalari E. Bronchopulmonary dysplasia. Am J Respir Crit Care Med 2001;163:1723-9.

15 Lougheed MD, Lemière C, Dell SD, et al. Canadian Thoracic Society Asthma Management Continuum - 2010 Consensus Summary for children six years of age and over, and adults. Can Respir J 2010;17:15-24.

16 Miller MR, Hankinson J, Brusasco V, et al. Standardisation of spirometry. Eur Respir J 2005;26:319-38. 
17 Macintyre N, Crapo RO, Viegi G, et al. Standardisation of the single-breath determination of carbon monoxide uptake in the lung. Eur Respir J 2005;26:720-35.

18 Wanger J, Clausen JL, Coates A, et al. Standardisation of the measurement of lung volumes. Eur Respir J 2005;26:511-22.

19 Quanjer PH, Stanojevic S, Cole TJ, et al. Multi-ethnic reference values for spirometry for the 3-95-yr age range: the global lung function 2012 equations. Eur Respir J 2012:40:1324-43.

20 Rosenthal M, Cramer D, Bain SH, et al. Lung function in White children aged 4 to 19 years: II--Single breath analysis and plethysmography. Thorax 1993;48:803-8.

21 American Thoracic Society ACOCP. ATS/ACCP Statement on cardiopulmonary exercise testing. Am J Respir Crit Care Med 2003;167:211-77.

22 Wasserman K, Hansen JE, Sue DY, et al. Principles of exercise testing and interpretation: including pathophysiology and clinical applications. Philadelphia: Lippincott Williams \& Wilkins, Wolters Kluwer Business, 2005.

23 Emerson SR, Kurti SP, Rosenkranz SK, et al. Decreased prevalence of exercise expiratory flow limitation from pre- to postpuberty. Med Sci Sports Exerc 2015;47:1503-11.

24 Borg GA. Psychophysical bases of perceived exertion. Med Sci Sports Exerc 1982;14:377-81.

25 Calverley PM, Koulouris NG. Flow limitation and dynamic hyperinflation: key concepts in modern respiratory physiology. Eur Respir J 2005;25:186-99.

26 O'Donnell DE, Voduc N, Fitzpatrick M, et al. Effect of salmeterol on the ventilatory response to exercise in chronic obstructive pulmonary disease. Eur Respir J 2004;24:86-94.

27 Oudiz RJ, Midde R, Hovenesyan A, et al. Usefulness of right-to-left shunting and poor exercise gas exchange for predicting prognosis in patients with pulmonary arterial hypertension. Am J Cardiol 2010;105:1186-91.

28 Robertson HT, Pellegrino R, Pini D, et al. Exercise response after rapid intravenous infusion of saline in healthy humans. J App/ Physiol 2004;97:697-703.
29 Bongers BC, Werkman MS, Takken T, et al. Ventilatory response to exercise in adolescents with cystic fibrosis and mild-to-moderate airway obstruction. Springerplus 2014;3:696.

30 Holland $A E$, Hill CJ, Jones $A Y$, et al. Breathing exercises for chronic obstructive pulmonary disease. Cochrane Database Syst Rev 2012;10:CD008250.

31 Chen $\mathrm{R}$, Chen $\mathrm{R}$, Chen $\mathrm{X}$, et al. Effect of endurance training on expiratory flow limitation and dynamic hyperinflation in patients with stable chronic obstructive pulmonary disease. Intern Med J 2014:44:791-800.

32 LoRusso TJ, Belman MJ, Elashoff JD, et al. Prediction of maximal exercise capacity in obstructive and restrictive pulmonary disease. Chest 1993;104:1748-54.

33 Babb TG, Viggiano R, Hurley $B$, et al. Effect of mild-to-moderate airflow limitation on exercise capacity. J Appl Physiol 1991;70:223-30.

34 Dimopoulou I, Tsintzas OK, Daganou M, et al. Contribution of lung function to exercise capacity in patients with chronic heart failure. Respiration 1999;66: 144-9.

35 Berntsen S, Lødrup Carlsen KC, Anderssen SA, et al. Factors associated with aerobic fitness in adolescents with asthma. Respir Med 2013;107:1164-71.

36 Clemm HH, Vollsæter M, Røksund OD, et al. Exercise capacity after extremely preterm birth. Development from adolescence to adulthood. Ann Am Thorac Soc 2014;11:537-45.

37 Kohansal R, Martinez-Camblor P, Agustí A, et al. The natural history of chronic airflow obstruction revisited: an analysis of the Framingham offspring cohort. Am J Respir Crit Care Med 2009;180:3-10.

38 Astrand I, Astrand PO, Hallbäck I, et al. Reduction in maximal oxygen uptake with age. J Appl Physiol 1973;35:649-54.

39 Thannickal VJ, Murthy M, Balch WE, et al. Blue Journal Conference. Aging and susceptibility to lung disease. Am J Respir Crit Care Med 2015;191:261-9.

40 Lovering AT, Elliott JE, Laurie SS, et al. Ventilatory and sensory responses in adult survivors of preterm birth and bronchopulmonary dysplasia with reduced exercise capacity. Ann Am Thorac Soc 2014;11:1528-37. 